\title{
Pharmacological activities of ginsenoside Rg5 (Review)
}

\author{
MING-YANG LIU ${ }^{1,2}$, FEI LIU ${ }^{3}$, YAN-LI GAO ${ }^{4}$, JIA-NING YIN ${ }^{4}$, WEI-QUN YAN ${ }^{5}$, JIAN-GUO LIU ${ }^{2}$ and HAI-JUN LI ${ }^{1}$ \\ ${ }^{1}$ Department of Immunity, Institute of Translational Medicine, Departments of ${ }^{2}$ Orthopaedics, \\ ${ }^{3}$ Obstetrics and ${ }^{4}$ Pediatrics, The First Hospital of Jilin University; ${ }^{5}$ Department of Tissue Engineering, \\ School of Pharmaceutical Sciences in Jilin University, Changchun, Jilin 130021, P.R. China
}

Received May 12, 2020; Accepted May 7, 2021

DOI: $10.3892 /$ etm.2021.10272

\begin{abstract}
Ginseng, a perennial plant belonging to genus Panax, has been widely used in traditional herbal medicine in East Asia and North America. Ginsenosides are the most important pharmacological component of ginseng. Variabilities in attached positions, inner and outer residues and types of sugar moieties may be associated with the specific pharmacological activities of each ginsenoside. Ginsenoside $\operatorname{Rg} 5$ ( $\operatorname{Rg} 5)$ is a minor ginsenoside synthesized during ginseng steaming treatment that exhibits superior pharmaceutical activity compared with major ginsenosides. With high safety and various biological functions, $\operatorname{Rg} 5$ may act as a potential therapeutic candidate for diverse diseases. To date, there have been no systematic studies on the activity of $\operatorname{Rg} 5$. Therefore, in this review, all available literature was reviewed and discussed to facilitate further research on $\mathrm{Rg} 5$.
\end{abstract}

\section{Contents}

1. Introduction

2. Pharmacological activities of $\operatorname{Rg} 5$

3. Conclusion

\section{Introduction}

Ginseng, a perennial plant belonging to genus Panax, has been widely used in traditional herbal medicine in east Asia and North America for millennia to reinforce immunity,

Correspondence to: Dr Hai-Jun Li, Department of Immunity, Institute of Translational Medicine, The First Hospital of Jilin University, 71 Xinmin Street, Changchun, Jilin 130021, P.R. China E-mail: hjli2012@jlu.edu.cn

Professor Jian-Guo Liu, Department of Orthopaedics, The First Hospital of Jilin University, 71 Xinmin Street, Changchun, Jilin 130021, P.R. China

E-mail: liujgjlu@163.com

Key words: Panax ginseng, ginsenoside $\mathrm{Rg} 5$, natural product, tumor, anti-inflammation provide nutrition and reduce fatigue $(1,2)$. Ginsenosides are unique triterpenoid saponins predominantly extracted from Panax ginseng C.A. Meyer that act as the main bioactive constituents of ginseng $(3,4)$. To date, $>100$ ginsenosides have been extracted from the roots, leaves, stems, fruits and flower heads of ginseng (3). All ginsenosides share a common four-ring hydrophobic structure, but differ in the number and types of glycosyl (5). Ginsenosides are classified into 20(S)-protopanaxadiol, 20(S)-protopanaxatriol saponins and oleanoic acid ginsenosides $(6,7)$. The variability of attached positions, inner and outer residues, and types of sugar moieties may be associated with the specific pharmacological activities of different ginsenosides (4). Glycosylated major ginsenosides, such as Rb1, Rb2, Rc, Rd, Re and Rg1, constitute $>80 \%$ of the total ginsenosides in various parts of ginseng (8). Deglycosylated minor ginsenosides, which have fewer sugar moieties attached on aglycon, are absent or present in smaller amounts in wild ginseng $(9,10)$.

Ginsenoside $\operatorname{Rg} 5$ (Rg5) is a minor ginsenoside synthesized during ginseng steaming treatment; the structural formula is displayed in Fig. 1 (11). It is obtained by the deglycosylation of ginsenoside $\mathrm{Rb} 1$ and dehydration of carbon at position 20 of ginsenoside $\operatorname{Rg} 3$, and exhibits superior pharmaceutical effect compared with major ginsenosides $(12,13)$. In previous studies, Rg5 was found to exert multiple pharmacological effects, such as antitumor, anti-inflammatory, antidiabetic, anti-osteoarthritis (OA), neuroprotective and cardioprotective properties (14-20). With high safety and various biological functions, $\operatorname{Rg} 5$ has the potential to act as a potential therapeutic candidate for diverse diseases. The present article reviewed the $\operatorname{Rg} 5$ literature and summarized its pharmacological activities.

\section{Pharmacological activities of $\mathbf{R g 5}$}

Anticancer effects. Cancer is a group of life-threatening diseases that are characterized by abnormal proliferation of cells with potential to invade and spread to surrounding and distant tissues. Conventional therapies for cancer include surgical resection, radiotherapy and chemotherapy $(21,22)$. Chemotherapy serves an important role in the treatment of malignant tumors (23). However, the severe side effects of traditional chemotherapy, such as myelosuppression and immune suppression, hinder the therapeutic effects (24). The use of ginsenosides as alternative antitumor agents has gained increasing attention $(25,26)$. Several studies have reported 
the antitumor effects on human gastric and breast cancer of $\mathrm{Rg} 5$, which are mainly associated with promoting apoptosis, autophagy and cell cycle arrest (15-18).

Breast cancer is a major health risk for the adult female population. The mechanism underlying the effects of Rg5 on breast cancer has been investigated in vivo and in vitro. Kim and Kim (15) demonstrated that Rg5 promoted breast cancer cell apoptosis via downregulation of the Bax/Bcl-2 pathway. It was also demonstrated that $\mathrm{Rg} 5$ inhibited breast cancer cell proliferation by arresting the cell cycle at the G0/G1 phase by promoting the expression of $\mathrm{p} 53$, p2 $1^{\mathrm{WAF} 1 / \mathrm{CIP} 1}$ and $\mathrm{p} 15^{\mathrm{INK} 4 \mathrm{~B}}$ and inhibiting the expression levels of cyclin D1, cyclin E2 and CDK4 (15). Moreover, it was discovered that Rg5 exhibited improved pro-apoptotic effects on human breast cancer cell lines compared with ginsenoside Rg3 (15). Zou and Liu (16) reported that Rg5 exhibited antiproliferative effects against breast cancer cells by activating the AMPK pathway. More recently, Liu et al (14) discovered that Rg5 induced breast cancer cell apoptosis and autophagy by inhibiting the PI3K/Akt/mTOR signaling pathway. In addition, Kim et al (13) demonstrated that $\operatorname{Rg} 5$ inhibited the growth of tumors in breast cancer mouse models by promoting tumor cell autophagy and apoptosis without damaging the normal functions of major organs and immune cells, which indicated that $\operatorname{Rg} 5$ exerts effects against breast cancer in vivo (17).

The effects and associated mechanisms underlying $\mathrm{Rg} 5$ in the treatment of digestive system cancer have also been reported. Liu and Fan (18) investigated the anticancer activity of ginsenoside in human gastric cancer cell lines and suggested that $\operatorname{Rg} 5$ inhibited cell proliferation by inducing G2/M phase arrest, apoptosis and autophagy by activating reactive oxygen species (ROS)-mediated MAPK pathways. Moreover, in a human xenograft nude mouse model, Rg5 displayed significant effects against gastric cancer with few side effects (18). Zhang et al (27) revealed that Rg5 suppressed proliferation and promoted the apoptosis of human esophageal cancer cells, which was associated with inhibition of the PI3K/Akt signaling pathway. Wang et al (28) demonstrated that $\mathrm{Rg} 5$ bound to annexin $\mathrm{A} 2$, and inhibited the interaction between annexin A2 and NF- $\mathrm{NB}$ p50 subunit, which resulted in the promotion of caspase activation and $\mathrm{NF}-\kappa \mathrm{B}$ activity in human hepatoma HepG2 cells. Lee et al (29) reported that Rg5 arrested the cell cycle of human hepatoma SK-HEP-1 cells at the G1/S transition phase through cyclin E-dependent protein kinase activity by increasing the expression of $\mathrm{p} 21^{\mathrm{Cip} / \mathrm{WAF} 1}$ and reducing cyclin $\mathrm{E}$.

Additionally, $\operatorname{Rg} 5$ promotes apoptosis in retinoblastoma cells by inhibiting the Akt signaling pathway and thereby downregulating Bcl-2 expression (30). Rg5 also promotes human cervical cancer cell apoptosis in concentration- and time-dependent manners by inducing DNA double-strand breaks and fragments (11). Rg5 has also been demonstrated to effectively overcome ATP binding cassette subfamily B member 1 (ABCB1)-mediated drug resistance by inhibiting ABCB1 transporter and blocking the activation of Akt/nuclear-related factor 2 pathways without affecting the expression of ABCB1 transporter (31). Therefore, $\operatorname{Rg} 5$ may serve as a chemosensitizer for reversing multidrug resistance.

With the development of nanomedicine, the antitumor effects of $\operatorname{Rg} 5$ through biocompatible nanoscale drug delivery systems has also been investigated (32). Dong et al (32) used folic acid (FA)-modified bovine serum albumin (BSA) nanoparticles (FA-Rg5-BSA NPs) as carriers to entrap Rg5. It was discovered that the FA-Rg5-BSA NPs exhibited superior anticancer activity compared with Rg5 in MCF-7 cells with low cytotoxicity to L929 cells. The FA-Rg5-BSA NPs facilitated cellular uptake and induced apoptosis in MCF-7 cells. Furthermore, an in vivo antitumor study demonstrated that FA-Rg5-BSA NPs were more effective in reducing tumor growth than Rg5 and Rg5-BSA NPs in an MCF-7 xenograft mouse model. This in vivo real-time bioimaging study demonstrated that the FA-Rg5-BSA NPs had an advanced ability to accumulate in tumors. These results indicated that FA-Rg5-BSA NPs has the potential to serve as an attractive therapeutic strategy for the management of cancer (32).

Anti-inflammatory effects. Inflammation is defined as a defensive mechanism characterized by the release of proinflammatory cytokines and the transmigration of inflammatory cells to protect human bodies from harmful stimuli $(19,20)$. Excessive or abnormal inflammatory responses have the potential to lead to various diseases, such as organ failure, central nerve system injury, tissue damage and even death (33). Thus, regulating the expression of inflammatory mediators should be beneficial for the treatment of inflammation-related diseases $(13,34)$. A number of studies have reported that Rg5 exerted anti-inflammatory effects in inflammatory responses by inhibiting the production of proinflammatory cytokines, such as TNF- $\alpha$ and IL- $1 \beta$ by interfering NF- $\kappa$ B or the NOD-, LRR- and pyrin domain-containing protein 3 (NLRP3) inflammasome pathways (35-40).

It has been demonstrated that $\mathrm{Rg} 5$ exhibited protective effects on major organs through its anti-inflammatory mechanisms. Kim et al (35) suggested that Rg5 ameliorates lung inflammation in mice by blocking the binding of lipopolysaccharide (LPS) to Toll-like receptor (TLR)-4 on macrophages, which are associated with inhibition of $\mathrm{NF}-\kappa \mathrm{B}$ activation. Park et al (36) demonstrated that $\operatorname{Rg} 5$ exerted protective effects against cisplatin-induced renal damage by attenuating JNK/p53/caspase-3 cascade-mediated inflammation. Li et al (37) demonstrated that $\mathrm{Rg} 5$ attenuated renal dysfunction by reducing the expression of inflammatory mediators, including NF- $\mathrm{B}$, p65 and cyclooxygenase-2 (COX-2). Similarly, Lee (38) reported that Rg5 also decreased the expression of $\mathrm{NF}-\kappa \mathrm{B}$, inducible nitric oxide (NO) synthase (iNOS) and COX-2 in HepG2 cells treated with TNF- $\alpha$ and thereby acted as a potential anti-inflammatory agent against hepatitis. The anti-inflammatory activity of Rg5 was increased when compared with ginsenoside Rb1, Rd and Rg3 and this increased bioactivity of $\operatorname{Rg} 5$ was hypothesized to be due to the higher lipophilicity compared with Rb1, Rd and Rg3 (38). Rg5 also ameliorated acetaminophen (APAP)-induced liver injury by suppressing APAP-induced expression of the inflammatory cytokines, TNF- $\alpha$ and IL-1 $\beta$ (39). Moreover, $\mathrm{Rg} 5$ demonstrated protective effects in high-fat diet/streptozotocin-induced diabetic nephropathy mice and improved renal injury by attenuating oxidative stress and inflammatory states by suppressing ROS-mediated activation of NLRP3 inflammasome, p38 MAPK and NF- $\mathrm{NB}$ signaling pathways in the kidneys of diabetic nephropathy mice (40). 


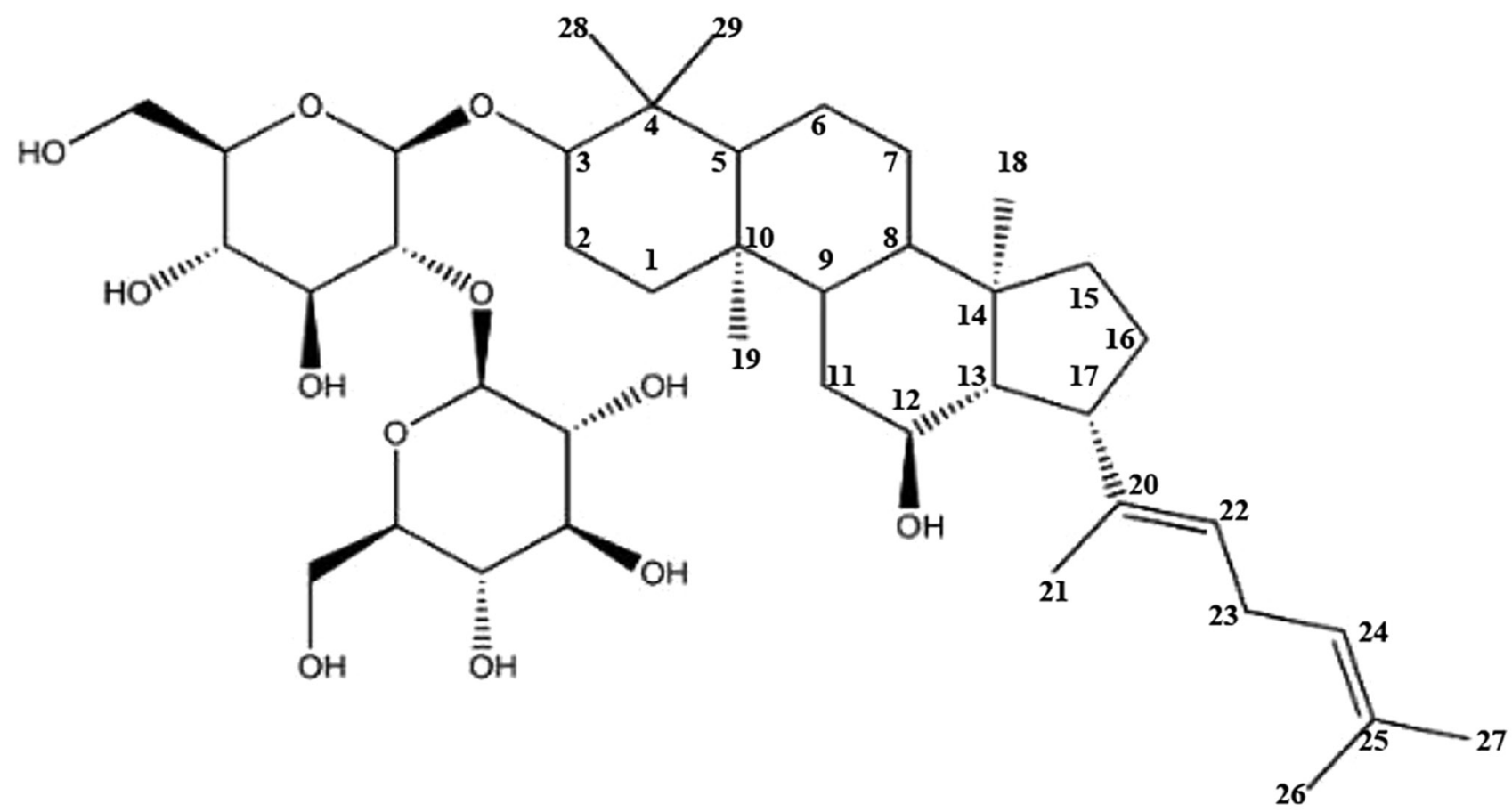

Figure 1. Structure of ginsenoside $\operatorname{Rg} 5$.

Sepsis is a systemic inflammatory response syndrome caused by the body's response to infection (41). High mobility group box 1 (HMGB1) is regarded as a crucial mediator of sepsis (42). The suppression of HMGB1-induced inflammatory reactions and maintenance of endothelial integrity have served as promising therapeutic strategies for the treatment of sepsis (43). Kim et al (44) demonstrated that Rg5 suppressed the release of HMGB1 in LPS-activated human umbilical vein endothelial cells (HUVECs). Moreover, $\operatorname{Rg} 5$ inhibited the adhesion and migration of leukocytes toward HUVECs. The aforementioned study indicated that $\operatorname{Rg} 5$ may be a potential therapeutic option for the treatment of severe vascular inflammatory diseases, such as sepsis and septic shock.

$\operatorname{Rg} 5$ has also demonstrated anti-inflammatory properties in dermal diseases $(45,46)$. Shin et al (45) demonstrated the inhibitory effects of $\mathrm{Rg} 5$ and its metabolite ginsenoside $\mathrm{Rh} 3$ in oxazolone-induced mouse ear contact dermatitis by inhibiting the expression of COX-2, TNF- $\alpha$ and IL- $1 \beta$ produced by macrophage cells and IFN- $\gamma$ produced by Thl cells. Ahn et al (46) discovered that Rg5 had anti-inflammatory effects in two atopic dermatitis-related cell lines. LPS-induced production of $\mathrm{NO}$ and ROS was downregulated by $\operatorname{Rg} 5$ in RAW264.7 cells, indicating that Rg5 has the ability to improve chronic inflammatory skin disease by blocking the NF-kB/p38/MAPK/STAT1 signaling pathways.

Anti-inflammatory effects of Rg5 in the central neural system have also been demonstrated. Chu et al (47) reported that $\operatorname{Rg} 5$ significantly suppressed the expression of pro-inflammation-related cytokines, including IL- $1 \beta, \mathrm{TNF}-\alpha, \mathrm{COX}-2$ and iNOS, and thereby attenuated neuroinflammatory responses in STZ-induced memory-impaired rats. $\operatorname{Rg} 5$ was also revealed to relieve cerebral ischemic injury by decreasing NF- $\kappa \mathrm{B}$ transcriptional activity and the expression of proinflammatory cytokines, such as IL- $1 \beta$, TNF- $\alpha$ and IL-6, by activating
TLR4/MyD88 and sirtuin 1 signaling pathways, contributing to reductions in cerebral ischemic injury (48). In addition, Lee et al (49) demonstrated that $\mathrm{Rg} 5$ suppressed neuroinflammation induced by LPS in BV2 microglial cells by inhibiting the MAPK and PI3K/Akt pathways.

Neuroprotective effects. Neurodegenerative diseases have become another category of health-threatening diseases (50). $\operatorname{Rg} 5$ exerts beneficial effects on nervous system diseases, such as Alzheimer's disease (AD) and Huntington's disease (HD) (51-53). AD is a multifactorial neurodegenerative disease featuring extracellular $\beta$-amyloid $(A \beta)$ plaques and intracellular neurofibrillary tangles in the brain (51). Inhibition of cAMP response element-binding protein (CREB) and brain-derived neurotrophic factor (BDNF) has the potential to lead to memory deficits in patients with AD $(52,53)$. Kim et al (54) revealed that $\operatorname{Rg} 5$ significantly reversed memory deficits induced by acetylcholinesterase using the passive avoidance, Y-maze and Morris water maze tasks in mice. The research revealed that treatment with Rg5 ameliorated the reduction of BDNF expression and CREB phosphorylation induced by scopolamine (54). Chu et al (47) demonstrated that $\mathrm{Rg} 5$ improves cognitive dysfunction in streptozotocin-induced AD rats by modulating the cholinergic system, decreasing $A \beta$ deposition and promoting the expression levels of neurotrophic factors BDNF and insulin-like growth factor 1 (IGF-1). Moreover, Rg5 has significant ameliorative effects on STZ-induced neuroinflammatory responses (47). Choi et al (55) revealed that $\operatorname{Rg} 5$ suppressed thermal stress-induced cell cycle arrest at G1/S phase by activating p21 and poly(ADP-ribose) polymerase cleavage. CREB and BDNF were also increased by Rg5 in thermal stress-exposed HT22 cells (55). HD is an autosomal-dominant neurogenic disorder that leads to progressive nerve cell damage in the brain. Wu et al (56) demonstrated Rg5 

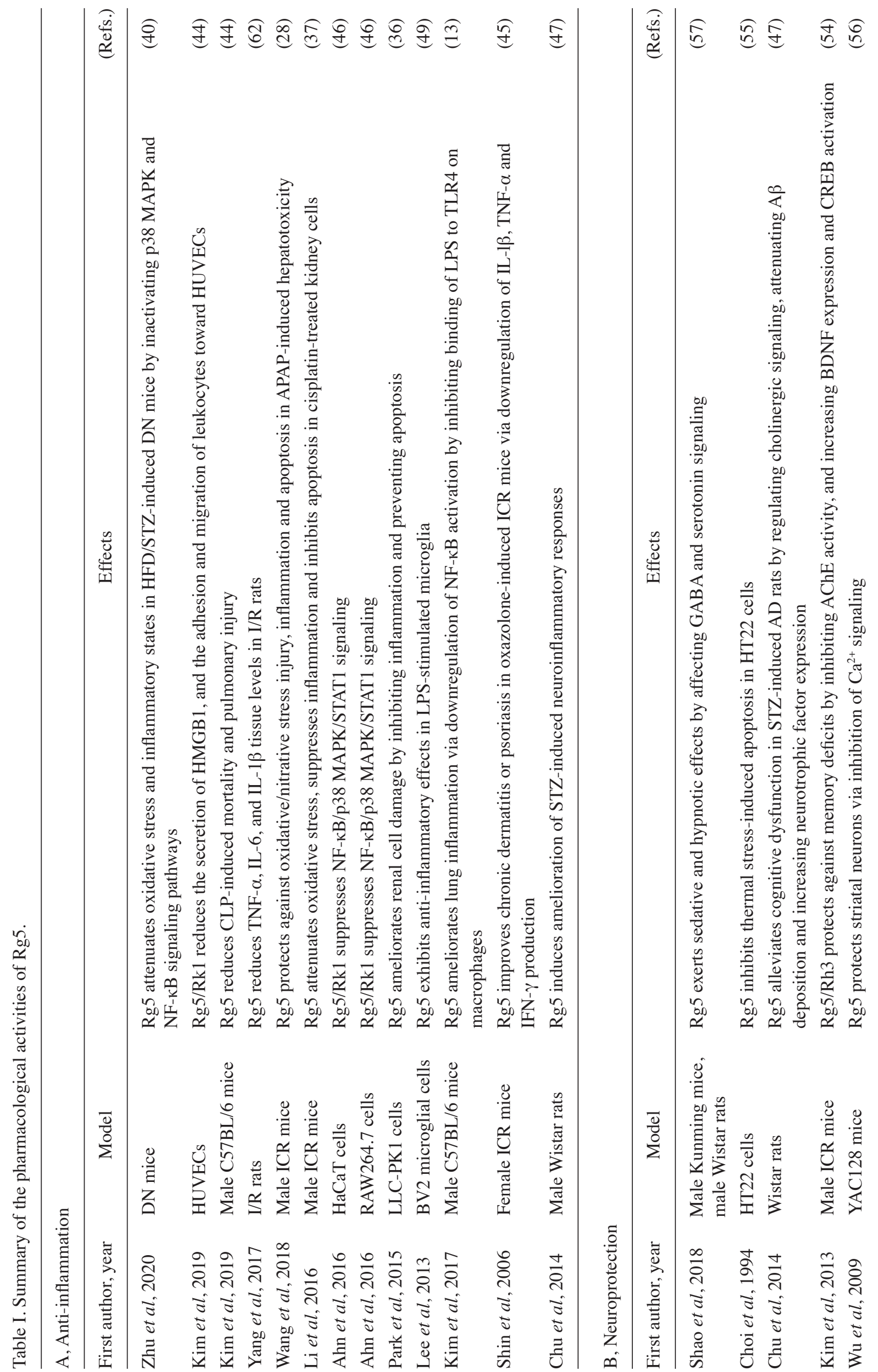


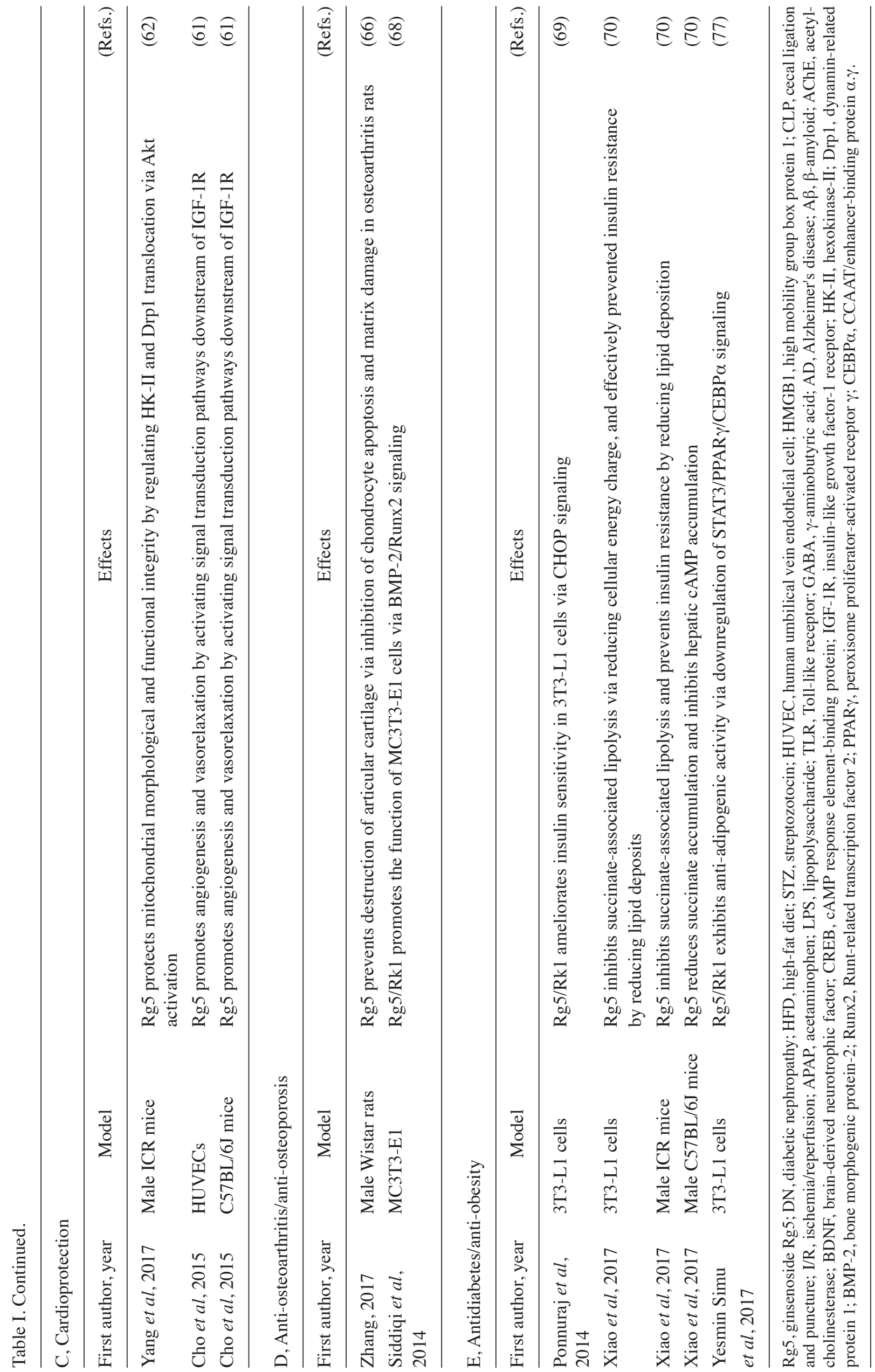




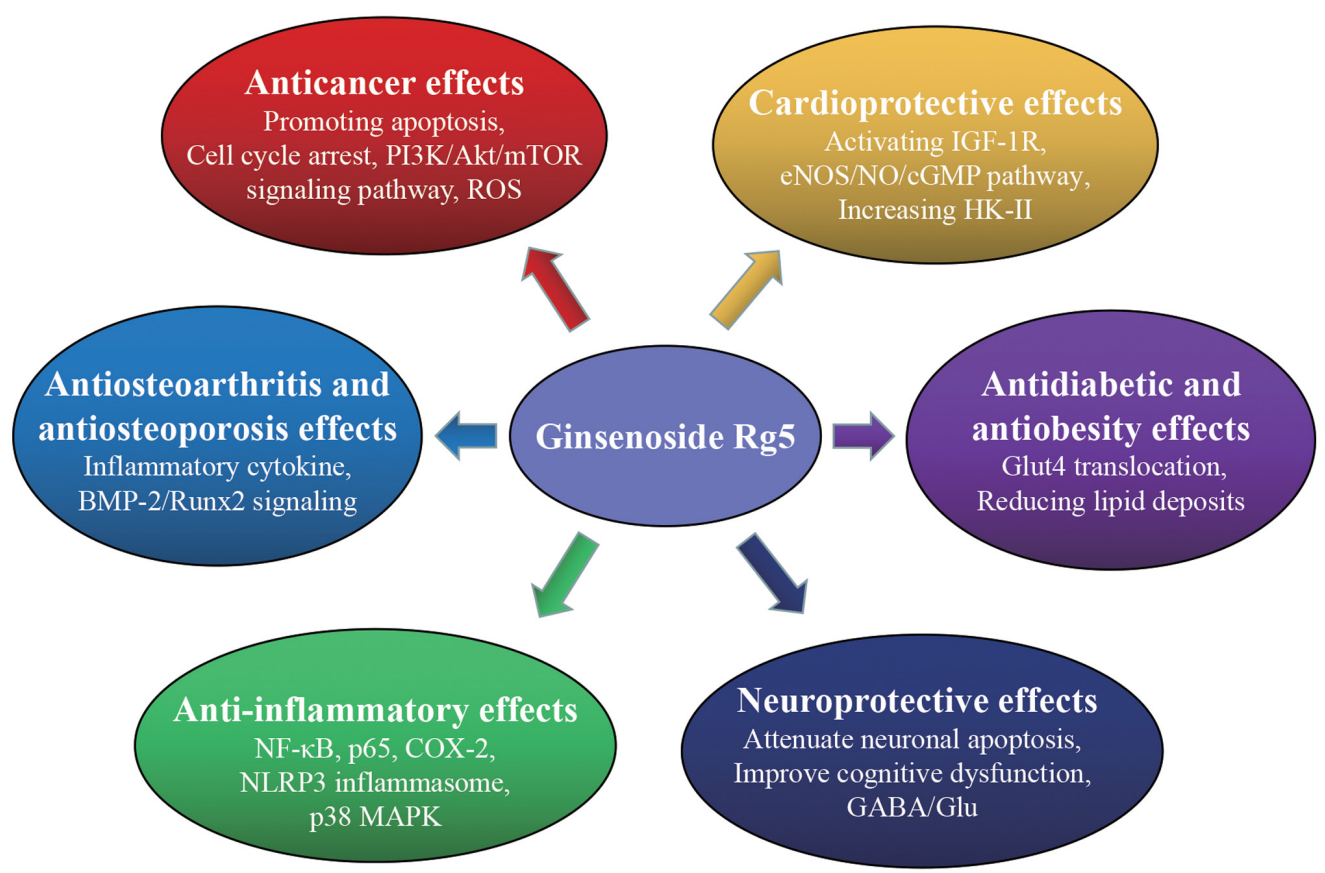

Figure 2. Potential mechanisms of pharmacological activities of ginsenoside Rg5. ROS, reactive oxygen species; IGF-1R, insulin-like growth factor; COX-2, cyclooxygenase-2; BMP-2, bone morphogenetic protein 2; Runx2, runt-related transcription factor 2; Glut4, glucose transporter 4; NO, nitric oxide; eNOS, endothelial NO synthase; HK-II, hexokinase-II; GABA, $\gamma$-aminobutyric acid; Glu, glutamate.

attenuates neuronal apoptosis by inhibiting glutamate-induced increases of $\mathrm{Ca}^{2+}$ concentrations in cultured medium spiny neurons, which indicated $\operatorname{Rg} 5$ has the potential to be useful for HD therapy. Wu et al (56) further discovered that the inhibitory activity of $\mathrm{Rg} 5$ on glutamate-induced $\mathrm{Ca}^{2+}$ responses was similar to ginsenoside Rc and far greater than ginsenoside Re.

In addition, an in vivo study revealed that $\operatorname{Rg} 5$ may regulate nerve transmission by affecting neurotransmitter and neuroregulatory receptors (57). Glutamate (Glu) is known as a major excitatory neurotransmitter, whereas $\gamma$-aminobutyric acid (GABA) is well-known as a major inhibitory neurotransmitter in the CNS $(58,59)$. Shao et al $(57)$ suggested that Rg5 downregulates the GABA/Glu ratio, and augments the expression of $\mathrm{GABA}_{\mathrm{A}}$ and $\mathrm{GABA}_{\mathrm{B}}$ receptors. Serotonin $(5-\mathrm{HT})$ is a neurotransmitter involved in sleep-wake cycle regulation (60). Shao et al (57) demonstrated that 5-hydroxytryptophan, together with a precursor of 5-HT, promotes the sleep effects of Rg5 in mice and Rg5 augments the expression of 5-HT1A. The results indicated that $\operatorname{Rg} 5$ exhibited hypotensive and sedative activities by modulating GABA and serotonin signaling in the nervous system, thus ameliorating sleep in mice models (57).

Cardioprotective effects. Studies into the therapeutic effects of $\operatorname{Rg} 5$ on cardiovascular diseases have also been reported. Cho et al (61) demonstrated that Rg5 regulates neovascularization and vasorelaxation by activating IGF-1 receptor (IGF-1R). The angiogenic activity of Rg5 is highly associated with a specific increase in IGF-1R phosphorylation and the subsequent activation of multiple angiogenic signals (61). Furthermore, the vasodilative activity of $\operatorname{Rg} 5$ is mediated by the endothelial NOS/NO/cGMP pathway (61). These findings offer a mechanistic explanation of the beneficial effects of Rg5 on neovascularization and endothelial function under pathological conditions. Yang et al (62) reported that Rg5 increased cardiomyocyte resistance to ischemic injury by regulating the translocation of two important enzymes, hexokinase-II (HK-II) and dynamin-related protein 1 (Drp1). Drp1 and HK-II exert opposite effects on mitochondrial function in cardiomyocytes by competing for binding to mitochondria (62). $\operatorname{Rg} 5$ protects mitochondrial morphological and functional integrity by suppressing Drp1 activation and increasing HK-II binding to cardiomyocyte mitochondria through Akt activation (62). These results provide a rationale for utilizing $\operatorname{Rg} 5$ for treating cardiovascular diseases.

Anti-OA and anti-osteoporosis (OP) effects. OA and OP are common bone diseases in middle-aged and elderly populations. OA involves a series of complicated processes characterized by the destruction of chondrocytes and remodeling of subchondral bones, resulting in progressive joint degeneration (63). The regulation of inflammatory cytokine networks by ginsenosides has attracted increased attention for the treatment of OA $(64,65)$. Zhang $(66)$ revealed that $\mathrm{Rg} 5$ prevented articular cartilage degradation and inhibited synovium disintegration in OA rat models. The level of OA-related enzyme metalloproteinase-13 decreased to $45 \%$ compared with controls; tissue inhibitors of metalloproteinase-1 increased by $67 \%$ after treatment with $\mathrm{Rg} 5$. The levels of inflammatory mediators, such as IL- $1 \beta$, TNF- $\alpha$, NO and iNOS, decreased by 67,54 , 32 and $49 \%$, respectively, after 1 month of treatment with Rg5 (66). The expression of bone morphogenetic protein-2 (BMP-2) and TGF- $\beta 1$ increased to 67 and 52\%, respectively, after treatment with Rg5. Therefore, Zhang (66) considered $\operatorname{Rg} 5$ useful for OA therapy.

OP systemically decreases bone mass and strength, and is characterized by the disturbance of osteoblast activity (67). Siddiqi et al (68) demonstrated that Rg5/Rk1 stimulates osteoblast cell growth and promotes the expression of 
osteoblastic markers, such as alkaline phosphatase activity and type I collagen content, BMP-2 and calcium deposition in dose-dependent manners. Moreover, Rg5/Rk1 also stimulates the mRNA expression of Runt-related transcription factor 2 (Runx2) and osteocalcin (68). These results indicate that $\mathrm{Rg} 5 / \mathrm{Rk} 1$ has the potential to prevent OP by stimulating osteoblast proliferation and differentiation via the BMP-2/Runx2 signaling pathway.

Antidiabetic and anti-obesity effects. The antidiabetic effect of $\mathrm{Rg} 5$ can be attributed to the amelioration of insulin resistance and reduction of glucagon response (67-72). A high level of insulin is required to regulate blood glucose under insulin resistance conditions. During endoplasmic reticulum stress, the $\mathrm{Rk} 1 / \mathrm{Rg} 5$ ginsenoside complex was found to improve insulin sensitivity and increase glucose uptake to exert protective effects in 3T3-L1 cells through CHOP-mediated glucose transporter 4 translocation (69). Furthermore, Xiao et al (71) discovered that Rg5 inhibited succinate-associated lipolysis by reducing cellular energy charge and effectively prevented insulin resistance in muscle by reducing lipid deposits. The inhibitory effects of $\mathrm{Rg} 5$ in hepatic glucagon response have also been demonstrated (70). Xiao et al (70) revealed that Rg5 decreased succinate accumulation by suppressing hepatic fatty acid oxidation and cAMP accumulation by blocking succinate/hypoxia-inducible factor- $1 \alpha$ expression, leading to an attenuated hepatic glucagon response.

Ginsenosides have also been widely reported to have an anti-obesity effect (73-76) and the anti-obesity effect of Rg5 has been reported in vitro. Yesmin Simu et al (77) demonstrated that Rg5/Rk1 inhibited lipid droplet accumulation and decreased triglyceride content in 3T3-L1 adipocyte cells. The expression levels of STAT3, peroxisome proliferator-activated receptor (PPAR) $\gamma$, CCAAT/enhancer-binding protein (CEBP) $\alpha$ and adaptor protein complex were also reduced in dose-dependent manners after treatment with $\mathrm{Rg} 5 / \mathrm{Rk} 1$. Furthermore, Yesmin Simu et al (77) reported no significant cytotoxicity effects on 3T3-L1 cells up to $100 \mu \mathrm{g} / \mathrm{ml}$. Their results indicated that $\operatorname{Rg} 5$ may have therapeutic potential for treating obesity via the STAT3/PPAR $\gamma / \mathrm{CEBP} \alpha$ signaling pathway.

\section{Conclusion}

The current review summarized the pharmacological effects of $\mathrm{Rg} 5$. In general, Rg5 has substantial potential activity for use as a broad-spectrum anticancer and anti-inflammatory drug. Rg5 has been reported to exert several positive effects on the nervous system, which potentiate the clinical applications of $\operatorname{Rg} 5$ in the treatment of neurodegenerative diseases. Additional studies have investigated other pharmacological properties, such as cardioprotective, anti-OA, anti-OP, antidiabetic and anti-obesity effects. The biological activities of Rg5 have been widely investigated and the mechanisms underlying the actions of $\operatorname{Rg} 5$ based on the existing studies are summarized in Table I and Fig. 2. These optimized therapies should also be evaluated for their efficacies in vivo. It may be possible to develop novel $\operatorname{Rg} 5$ analogues with improved efficacy, pharmacokinetics and bioavailability profiles. Evidence of Rg5 efficacy has yet to be demonstrated in humans. There is a significant need to perform larger cohort clinical studies to confirm Rg5 efficacy for improved applications in the clinic. With this considered, further investigations in clinical trials are highly recommended to provide more reliable evidence for the clinical efficacy of $\operatorname{Rg} 5$.

\section{Acknowledgements}

Not applicable.

\section{Funding}

This study was supported in part by the National Natural Science Foundation of China (grant nos. 81970529 and 81700018), the Jilin Provincial Natural Science Foundation of China (grant no. 20180101135JC), the Jilin Provincial Finance Foundation (grant no. 2018SCZWSZX-043), Jilin Provincial Health Commission Foundation (grant no. 2018Q020), the Interdisciplinary Chemistry and Medicine Foundation of Jilin University (grant no. JDYYJCHX004), the Norman Bethune Program of Jilin University (grant no. 2019007) and the Foundation for The Excellent Youth Scholars of Jilin University.

\section{Availability of data and materials}

Not applicable.

\section{Authors' contributions}

MYL and FL wrote the manuscript. YLG and JNY collected the references and produced the figure. HJL designed, interpreted and funded the study and revised the manuscript. WQY and JGL revised the manuscript. All authors read and approved the final version of the manuscript.

\section{Ethics approval and consent to participate}

Not applicable.

\section{Patient consent for publication}

Not applicable.

\section{Competing interests}

The authors declare that they have no competing interests.

\section{References}

1. De Souza LR, Jenkins AL, Sievenpiper JL, Jovanovski E, Rahelić D and Vuksan V: Korean red ginseng (Panax ginseng C.A. Meyer) root fractions: Differential effects on postprandial glycemia in healthy individuals. J Ethnopharmacol 137: 245-250, 2011.

2. Zhou QL, Zhu DN, Yang YF, Xu W and Yang XW: Simultaneous quantification of twenty-one ginsenosides and their three aglycones in rat plasma by a developed UFLC-MS/MS assay: Application to a pharmacokinetic study of red ginseng. J Pharm Biomed Anal 137: 1-12, 2017.

3. Christensen LP: Ginsenosides chemistry, biosynthesis, analysis, and potential health effects. Adv Food Nutr Res 55: 1-99, 2009.

4. Shi W, Wang Y, Li J, Zhang $\mathrm{H}$ and Ding L: Investigation of ginsenosides in different parts and ages of Panax ginseng. Food Chem 102: 664-668, 2007. 
5. Chen RJ, Chung TY, Li FY, Lin NH and Tzen JTC: Effect of sugar positions in ginsenosides and their inhibitory potency on Na+/K+-ATPase activity. Acta Pharmacol Sin 30: 61-69, 2009.

6. Shin BK, Kwon SW and Park JH: Chemical diversity of ginseng saponins from Panax ginseng. J Ginseng Res 39: 287-298, 2015.

7. Shin KC and Oh DK: Classification of glycosidases that hydrolyze the specific positions and types of sugar moieties in ginsenosides. Crit Rev Biotechnol 36: 1036-1049, 2016.

8. Jin S, Jeon JH, Lee S, Kang WY, Seong SJ, Yoon YR, Choi MK and Song IS: Detection of 13 ginsenosides (Rb1, Rb2, Rc, Rd, Re Rf, Rg1, Rg3, Rh2, F1, compound K, 20(S)-protopanaxadiol, and 20(S)-protopanaxatriol) in human plasma and application of the analytical method to human pharmacokinetic studies following two week-repeated administration of red ginseng extract. Molecules 24: 2618, 2019.

9. Cui CH, Jeon BM, Fu Y, Im WT and Kim SC: High-density immobilization of a ginsenoside-transforming $\beta$-glucosidase for enhanced food-grade production of minor ginsenosides. Appl Microbiol Biotechnol 103: 7003-7015, 2019.

10. Noh KH and Oh DK: Production of the rare ginsenosides compound $\mathrm{K}$, compound $\mathrm{Y}$, and compound Mc by a thermostable beta-glycosidase from sulfolobus acidocaldarius. Biol Pharm Bull 32: 1830-1835, 2009.

11. Liang LD, He T, Du TW, Fan YG, Chen DS and Wang Y: Ginsenoside-Rg5 induces apoptosis and DNA damage in human cervical cancer cells. Mol Med Rep 11: 940-946, 2015.

12. Qi LW, Wang CZ and Yuan CS: Ginsenosides from American ginseng: Chemical and pharmacological diversity. Phytochemistry 72: 689-699, 2011.

13. Kim JH, Yi YS, Kim MY and Cho JY: Role of ginsenosides, the main active components of Panax ginseng, in inflammatory responses and diseases. J Ginseng Res 41: 435-443, 2017.

14. Liu Y and Fan D: The preparation of ginsenoside Rg5, its antitumor activity against breast cancer cells and its targeting of PI3K. Nutrients 12: 246, 2020.

15. Kim SJ and Kim AK: Anti-breast cancer activity of fine black ginseng (Panax ginseng Meyer) and ginsenoside Rg5. J Ginseng Res 39: 125-134, 2015.

16. Zou Y and Liu P: Ginsenoside-Rg5 inhibits proliferation of the breast carcinoma cells through promotion of the proteins involved in AMP kinase pathway. Int J Clin Exp Med 9: 17664-17669, 2016.

17. Liu Y and Fan D: Ginsenoside Rg5 induces apoptosis and autophagy via the inhibition of the PI3K/Akt pathway against breast cancer in a mouse model. Food Funct 9: 5513-5527, 2018.

18. Liu Y and Fan D: Ginsenoside Rg5 induces G2/M phase arrest, apoptosis and autophagy via regulating ROS-mediated MAPK pathways against human gastric cancer. Biochem Pharmacol 168 : 285-304, 2019.

19. Jin HS, Suh HW, Kim SJ and Jo EK: Mitochondrial control of innate immunity and inflammation. Immune Netw 17: 77-88, 2017.

20. Medzhitov R: Origin and physiological roles of inflammation. Nature 454: 428-435, 2008

21. Harrison DJ, Geller DS, Gill JD, Lewis VO and Gorlick R: Current and future therapeutic approaches for osteosarcoma. Expert Rev Anticancer Ther 18: 39-50, 2018.

22. Akram M, Iqbal M, Daniyal M and Khan AU: Awareness and current knowledge of breast cancer. Biol Res 50: 33, 2017.

23. Sun M, Ye Y, Xiao L, Duan X, Zhang Y and Zhang H: Anticancer effects of ginsenoside $\operatorname{Rg} 3$ (Review). Int J Mol Med 39: 507-518, 2017.

24. Baldo BA and Pham NH: Adverse reactions to targeted and non-targeted chemotherapeutic drugs with emphasis on hypersensitivity responses and the invasive metastatic switch. Cancer Metastasis Rev 32: 723-761, 2013.

25. Majeed F, Malik FZ, Ahmed Z, Afreen A, Afzal MN and Khalid N: Ginseng phytochemicals as therapeutics in oncology: Recent perspectives. Biomed Pharmacother 100: 52-63, 2018.

26. Nakhjavani M, Hardingham JE, Palethorpe HM, Tomita Y, Smith E, Price TJ and Townsend AR: Ginsenoside Rg3: Potential molecular targets and therapeutic indication in metastatic breast cancer. Medicines (Basel) 6: 17, 2019.

27. Zhang D, Wang A, Feng J, Zhang Q, Liu L and Ren $\mathrm{H}$ : Ginsenoside Rg5 induces apoptosis in human esophageal cancer cells through the phosphoinositide-3 kinase/protein kinase B signaling pathway. Mol Med Rep 19: 4019-4026, 2019.

28. Wang YS, Li H, Li Y, Zhu H and Jin YH: Identification of natural compounds targeting annexin A2 with an anti-cancer effect. Protein Cell 9: 568-579, 2018.
29. Lee KH, Lee YH, Kim SI, Park JH and Lee SK: Ginsenoside-Rg5 suppresses cyclin E-dependent protein kinase activity via up-regulating p21Cip/WAF1 and down-regulating cyclin $\mathrm{E}$ in SK-HEP-1 cells. Anticancer Res 17: 1067-1072, 1997.

30. Cui Y, Su Y, Deng L and Wang W: Ginsenoside-Rg5 inhibits retinoblastoma proliferation and induces apoptosis through suppressing BCL2 expression. Chemotherapy 63: 293-300, 2018.

31. Feng SL, Luo HB, Cai L, Zhang J, Wang D, Chen YJ, Zhan HX, Jiang ZH and Xie Y: Ginsenoside Rg5 overcomes chemotherapeutic multidrug resistance mediated by ABCB1 transporter: In vitro and in vivo study. J Ginseng Res 44: 247-257, 2020.

32. Dong Y, Fu R, Yang J, Ma P, Liang L, Mi Y and Fan D: Folic acid-modified ginsenoside Rg5-loaded bovine serum albumin nanoparticles for targeted cancer therapy in vitro and in vivo. Int J Nanomedicine 14: 6971-6988, 2019.

33. Bortolotti P, Faure E and Kipnis E: Inflammasomes in tissue damages and immune disorders after trauma. Front Immunol 9: 1900, 2018

34. Yi YS: Roles of ginsenosides in inflammasome activation. J Ginseng Res 43: 172-178, 2019.

35. Kim TW, Joh EH, Kim B and Kim DH: Ginsenoside Rg5 ameliorates lung inflammation in mice by inhibiting the binding of LPS to toll-like receptor-4 on macrophages. Int Immunopharmacol 12: 110-116, 2012.

36. Park JY, Choi P, Kim T, Ko H, Kim HK, Kang KS and Ham J: Protective effects of processed ginseng and its active ginsenosides on cisplatin-induced nephrotoxicity: In vitro and in vivo studies. J Agric Food Chem 63: 5964-5969, 2015.

37. Li W, Yan M, Liu Y, Liu Z, Wang Z, Chen C, Zhang J and Sun YS: Ginsenoside Rg5 ameliorates cisplatin-induced nephrotoxicity in mice through inhibition of inflammation, oxidative stress, and apoptosis. Nutrients 8: 566, 2016.

38. Lee SM: Anti-inflammatory effects of ginsenosides Rg5, Rz1, and Rk1: Inhibition of TNF- $\alpha$-induced NF- $\kappa \mathrm{B}, \mathrm{COX}-2$, and iNOS transcriptional expression. Phytother Res 28: 1893-1896, 2014.

39. Wang Z, Hu JN, Yan MH, Xing JJ, Liu WC and Li W: Caspase-mediated anti-apoptotic effect of ginsenoside Rg5, a main rare ginsenoside, on acetaminophen-induced hepatotoxicity in mice. J Agric Food Chem 65: 9226-9236, 2017.

40. Zhu Y, Zhu C, Yang H, Deng J and Fan D: Protective effect of ginsenoside Rg5 against kidney injury via inhibition of NLRP3 inflammasome activation and the MAPK signaling pathway in high-fat diet/streptozotocin-induced diabetic mice. Pharmacol Res 155: 104746, 2020.

41. Cecconi M, Evans L, Levy M and Rhodes A: Sepsis and septic shock. Lancet 392: 75-87, 2018.

42. Andersson U, Wang H, Palmblad K, Aveberger AC, Bloom O, Erlandsson-Harris H, Janson A, Kokkola R, Zhang M, Yang H, and Tracey KJ: High mobility group 1 protein (HMG-1) stimulates proinflammatory cytokine synthesis in human monocytes. J Exp Med 192: 565-570, 2000.

43. Bae JS: Role of high mobility group box 1 in inflammatory disease: Focus on sepsis. Arch Pharm Res 35: 1511-1523, 2012.

44. Kim JE, Lee W, Yang S, Cho SH, Baek MC, Song GY and Bae JS: Suppressive effects of rare ginsenosides, Rk1 and Rg5, on HMGB1-mediated septic responses. Food Chem Toxicol 124: $45-53,2019$

45. Shin YW, Bae EA and Kim DH: Inhibitory effect of ginsenoside $\mathrm{Rg} 5$ and its metabolite ginsenoside Rh3 in an oxazolone-induced mouse chronic dermatitis model. Arch Pharm Res 29: 685-690, 2006.

46. Ahn S, Siddiqi MH, Aceituno VC, Simu SY, Zhang J, Jimenez Perez ZE, Kim YJ and Yang DC: Ginsenoside Rg5:Rk1 attenuates TNF- $\alpha / \mathrm{IFN}-\gamma$-induced production of thymusand activation-regulated chemokine (TARC/CCL17) and LPS-induced NO production via downregulation of NF- $\kappa \mathrm{B} / \mathrm{p} 38$ MAPK/STAT1 signaling in human keratinocytes and macrophages. In Vitro Cell Dev Biol Anim 52: 287-295, 2016.

47. Chu S, Gu J, Feng L, Liu J, Zhang M, Jia X, Liu M and Yang D: Ginsenoside Rg5 improves cognitive dysfunction and beta-amyloid deposition in STZ-induced memory impaired rats via attenuating neuroinflammatory responses. Int Immunopharmacol 19: 317-326, 2014.

48. Cheng Z, Zhang M, Ling C, Zhu Y, Ren H, Hong C, Qin J, Liu T and Wang J: Neuroprotective effects of ginsenosides against cerebral ischemia. Molecules 24: 1102, 2019.

49. Lee YY,Park JS, Jung JS, Kim DH andKimHS: Anti-inflammatory effect of ginsenoside Rg5 in lipopolysaccharide-stimulated BV2 microglial cells. Int J Mol Sci 14: 9820-9833, 2013. 
50. Liu FF, Zhang Z, Chen W, Gu HY and Yan QJ: Regulatory mechanism of microRNA-377 on CDH13 expression in the cell model of Alzheimer's disease. Eur Rev Med Pharmacol Sci 22: 2801-2808, 2018.

51. Bolognin S, Blanchard J, Wang X, Basurto-Islas G, Tung YC, Kohlbrenner E, Grundke-Iqbal I and Iqbal K: An experimental rat model of sporadic Alzheimer's disease and rescue of cognitive impairment with a neurotrophic peptide. Acta Neuropathol 123 : 133-151, 2012.

52. Tao X, Finkbeiner S, Arnold D, Shaywitz A and Greenberg M: $\mathrm{Ca}^{2+}$ influx regulates BDNF transcription by a CREB family transcription factor-dependent mechanism. Neuron 20: 709-726, 1998.

53. Bourtchuladze R, Frenguelli B, Blendy J, Cioffi D, Schutz G and Silva A: Deficient long-term memory in mice with a targeted mutation of the cAMP-responsive element-binding protein. Cell 79: 59-68, 1994.

54. Kim EJ, Jung IH, Van Le TK, Jeong JJ, Kim NJ and Kim DH: Ginsenosides Rg5 and Rh3 protect scopolamine-induced memory deficits in mice. J Ethnopharmacol 146: 294-299, 2013.

55. Choi SY, Kim KJ, Song JH and Lee BY: Ginsenoside Rg5 prevents apoptosis by modulating heme-oxygenase-1/nuclear factor E2-related factor 2 signaling and alters the expression of cognitive impairment-associated genes in thermal stress-exposed HT22 cells. J Ginseng Res 42: 225-228, 2018.

56. Wu J, Jeong HK, Bulin SE, Kwon SW, Park JH and Bezprozvanny I: Ginsenosides protect striatal neurons in a cellular model of Huntington's disease. J Neurosci Res 87: 1904-1912, 2009.

57. Shao J, Zheng X, Qu L, Zhang H, Yuan H, Hui J, Mi Y, Ma P and Fan D: Ginsenoside Rg5/Rk1 ameliorated sleep via regulating the GABAergic/serotoninergic signaling pathway in a rodent model. Food Funct 11: 1245-1257, 2020.

58. Yuan CS, Attele AS, Wu JA and Liu D: Modulation of American ginseng on brainstem GABAergic effects in rats. J Ethnopharmacol 62: 215-222, 1998.

59. Sattler R and Tymianski M: Molecular mechanisms of glutamate receptor-mediated excitotoxic neuronal cell death. Mol Neurobiol 24: 107-129, 2001.

60. Yuan Q, Joiner WJ and Sehgal A: A sleep-promoting role for the Drosophila serotonin receptor 1A. Curr Biol 16: 1051-1062, 2006.

61. Cho YL, Hur SM, Kim JY, Kim JH, Lee DK, Choe J, Won MH, Ha KS, Jeoung D, Han S, et al: Specific activation of insulin-like growth factor-1 receptor by ginsenoside $\mathrm{Rg} 5$ promotes angiogenesis and vasorelaxation. J Biol Chem 290: 467-477, 2015.

62. Yang YL, Li J, Liu K, Zhang L, Liu Q, Liu B and Qi LW: Ginsenoside $\operatorname{Rg} 5$ increases cardiomyocyte resistance to ischemic injury through regulation of mitochondrial hexokinase-II and dynamin-related protein 1. Cell Death Dis 8: e2625, 2017.

63. Wojdasiewicz P, Poniatowski $€$ and Szukiewicz D: The role of inflammatory and anti-inflammatory cytokines in the pathogenesis of osteoarthritis. Mediators Inflamm 2014: 561459, 2014.

64. Cheng W, Jing J, Wang Z, Wu D and Huang Y: Chondroprotective effects of ginsenoside Rg1 in human osteoarthritis chondrocytes and a rat model of anterior cruciate ligament transection. Nutrients 9: 263, 2017.
65. Chen Y, Lin S, Sun Y, Pan X, Xiao L, Zou L, Ho KW and Li G: Translational potential of ginsenoside $\mathrm{Rb} 1$ in managing progression of osteoarthritis. J Orthop Translat 6: 27-33, 2016.

66. Zhang P: Ginsenoside-Rg5 treatment inhibits apoptosis of chondrocytes and degradation of cartilage matrix in a rat model of osteoarthritis. Oncol Rep 37: 1497-1502, 2017.

67. Wang T, Liu Q, Tjhioe W, Zhao J, Lu A, Zhang G, Tan RX, Zhou M, Xu J and Feng HT: Therapeutic potential and outlook of alternative medicine for osteoporosis. Current Drug Targets 18: 1051-1068, 2017.

68. Siddiqi MH, Siddiqi MZ, Ahn S, Kang S, Kim YJ, Veerappan K, Yang DU and Yang DC: Stimulative effect of ginsenosides Rg5:Rk1 on murine osteoblastic MC3T3-E1 cells. Phytother Res 28: 1447-1455, 2014.

69. Ponnuraj SP, Siraj F, Kang S, Noh HY, Min JW, Kim YJ and Yang DC: Amelioration of insulin resistance by Rk1 + Rg5 complex under endoplasmic reticulum stress conditions. Pharmacognosy Res 6: 292-296, 2014

70. Xiao N, Lou MD, Lu YT, Yang LL, Liu Q, Liu B Qi LW and Li P: Ginsenoside Rg5 attenuates hepatic glucagon response via suppression of succinate-associated HIF-1 $\alpha$ induction in HFD-fed mice. Diabetologia 60: 1084-1093, 2017.

71. Xiao N, Yang LL, Yang YL, Liu LW, Li J, Liu B, Liu K, Qi LW and Li P: Ginsenoside Rg5 inhibits succinate-associated lipolysis in adipose tissue and prevents muscle insulin resistance. Front Pharmacol 8: 43, 2017.

72. Bai L, Gao J, Wei F, Zhao J, Wang D and Wei J: Therapeutic potential of ginsenosides as an adjuvant treatment for diabetes. Front Pharmacol 9: 423, 2018.

73. Park HJ, Kim JH and Shim I: Anti-obesity effects of ginsenosides in high-fat diet-fed rats. Chin J Integr Med 25: 895-901, 2019.

74. Liu H, Liu M, Jin Z, Yaqoob S, Zheng M, Cai D, Liu J and Guo S: Ginsenoside Rg2 inhibits adipogenesis in 3T3-L1 preadipocytes and suppresses obesity in high-fat-diet-induced obese mice through the AMPK pathway. Food Funct 10: 3603-3614, 2019.

75. Liu H, Wang J, Liu M, Zhao H, Yaqoob S, Zheng M, Cai D and Liu J: Antiobesity effects of ginsenoside Rg1 on 3T3-L1 preadipocytes and high fat diet-induced obese mice mediated by AMPK. Nutrients 10: 830, 2018.

76. Zhang L, Zhang L, Wang X and Si H: Anti-adipogenic effects and mechanisms of ginsenoside Rg3 in pre-adipocytes and obese mice. Front Pharmacol 8: 113, 2017.

77. Yesmin Simu S, Ahn S, Castro-Aceituno V and Yang DC: Ginsenoside Rg5: Rk1 exerts an anti-obesity effect on 3T3-L1 Cell Line by the downregulation of PPAR $\gamma$ and CEBP $\alpha$. Iran J Biotechnol 15: 252-259, 2017.

This work is licensed under a Creative Commons Attribution-NonCommercial-NoDerivatives 4.0 International (CC BY-NC-ND 4.0) License. 